\title{
Reversal of SARS-CoV2 Induced Hypoxia by Nebulized Sodium-Ibuprofen in a Compassionate Use Program
}

\section{Oscar Salva}

Clinica Independencia

\section{Pablo Alexis Doreski}

Fundación Respirar: Fundacion Respirar

\section{Celia Sara Giler}

Clinica Independencia

\section{Dario Conrado Quinodoz}

Sanatorio de la Cañada Villa Maria

\section{Lucia Guadalupe Guzman}

Sanatorio de la Cañada, Villa Maria

\section{Sonia Edith Munoz}

Instituto de Investigaciones en Ciencias de la Salud

\section{Mariana N Carrillo}

Instituto de Investigaciones en Clencias de la Salud

\section{Daniela Josefina Porta}

Instituto de Investigaciones en Clencias de la Salud

German Ambasch

Sanatorio Mayo Privado SA

\section{Esteban Coscia}

Sanatorio Mayo Privado SA

Jorge Luis Tambini Diaz

Sanatorio Mayo Privado SA

\section{German David Bueno}

Sanatorio Mayo Privado SA

Jorge Oscar Fandi

Clinica Independencia

Miriam Angelica Maldonado

Hospital San Roque

\section{Leandro Eugenio Peña Chiappero}

Hospital San Roque

\section{Fernando Fournier}


Clinica Francesa

\section{Hernan Alejandro Pérez}

Sanatorio Alive

\section{Mauro Andres Quiroga}

Instituto Modelo de Cardiologia

Javier Agustin Sala Mercado

Instituto Modelo de Cardiologia

Carlos Alberto Martínez Picco

Clinica del Sol

\section{Luis Alberto Argañaras}

Quimica Luar SRL

Nicolas Martinez Rios

Quimica Luar SRL

\section{Galia I. Kalayan}

Quimica Luar SRL

\section{Dante Miguel Beltramo}

CEPROCOR: Centro de Excelencia en Productos y Procesos Cordoba

Nestor H Garcia ( garcia_nestor@hotmail.com )

Instituto de Investigación en Ciencias de la Salud https://orcid.org/0000-0002-9057-9030

\section{Research}

Keywords: COVID-19, SARS-CoV-2, Coronavirus, hypoxemia, acute respiratory distress syndrome, viral pneumonia

Posted Date: April 13th, 2021

DOI: https://doi.org/10.21203/rs.3.rs-390980/v1

License: (c) (i) This work is licensed under a Creative Commons Attribution 4.0 International License. Read Full License 


\section{Abstract}

\section{Background}

Sodium-ibuprofenate in hypertonic saline (NalHS) administered directly to the lungs by nebulization and inhalation has antibacterial and anti-inflammatory effects with the potential to deliver these benefits to hypoxic patients. We describe a compassionate use program that offered this therapy to hospitalized COVID-19 patients.

\section{Methods}

NalHS (50 mg ibuprofen, tid) was provided in addition to standard of care to hospitalized Covid-19 patients until oxygen saturation levels of $>94 \%$ were achieved on ambient air. Patients wore a containment hood to diminish aerosolization. Outcome data from participating patients treated at multiple hospitals in Argentina between April 04, 2020, through October 31, 2020 are summarized.

\section{Results}

383 patients were treated, including 327 not on mechanical ventilation at baseline (MV) and 56 ICU

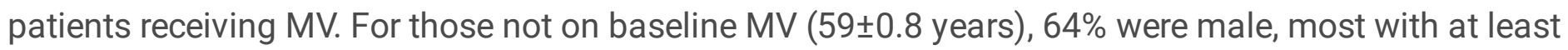
one recognized risk factor for disease severity, and mean NEWS2 score prior to treatment initiation of 7.0 \pm 0.1 . The average length of stay (ALOS) was $11.5 \pm 0.3$ days and length of treatment (LOT) $9.0 \pm 0.2$ days. In patients on baseline MV (60.6 \pm 2.2 years), $69.9 \%$ were male, baseline mean NEWS2 Score was $8.8 \pm 0.4$, ALOS $15.5 \pm 1.4$ days and LOT $10.5 \pm 0.7$ days. Reversal of deterioration in oxygenation and NEWS2 scores was observed acutely following initiation of therapy. Overall in-hospital mortality was $10.7 \%$ among patients not on MV at baseline, and 19.6\% among patients receiving MV at baseline. No serious adverse events were considered related to ibuprofen therapy.

\section{Conclusions}

Treatment of COVID-19 pneumonitis with inhalational nebulized NalHS was associated with rapid improvement in hypoxia and vital signs, with no serious adverse events attributed to therapy. Nebulized NalHS is worthy of further study in randomized, placebo-controlled trials.

(ClinicalTrials.gov:NCT04382768).

\section{Background}

Disease due to severe acute respiratory syndrome coronavirus 2 (SARS CoV-2), COVID-19, has taken the world by storm. As of this writing, an estimated 87 million cases and nearly 2 million deaths have been recorded by the World Health Organization (1) and effective therapy options remain limited. To date, dexamethasone and monoclonal antibody therapies have demonstrated benefit in patients with severe illness (2), while evidence of benefit for many other drugs remains unknown (3) or disproven (4-7). 
A hallmark of severe COVID-19 is profound hypoxia, which may progress to acute respiratory distress syndrome (ARDS). Clinically, these changes are accompanied by characteristic ground glass infiltrates on chest tomography and significant hypoxia (8). In autopsy series, several notable features were described, including diffuse alveolar damage with perivascular T-cell infiltration; severe endothelial injury with intracellular virus, widespread thrombosis with microangiopathy and intussusceptive angiogenesis and pathological vascular dilation in diseased area of lung (9).

In severe COVID-19, local release of autacoids leading to vascular injury and permeability results in excess alveolar fluid protein accumulation. This phenomenon, coupled with vasodilation and intrapulmonary shunting of non-oxygenated blood has been characterized as a 'bradykinin storm' (10) based on the observation of reduced ACE expression (with corresponding local loss of vasoconstriction), while ACE2, bradykinin and kallikrein expression were noted to be increased in diseased tissues. This mismatch contributes to ventilation/perfusion mismatch and resulting hypoxia. This hypothesis has been supported by demonstration of intrapulmonary shunting in severe COVID-19 (13). Importantly, ibuprofen can prevent bradykinin induced inflammation at the respiratory mucosa (11) and ibuprofen has also been demonstrated to prevent bradykinin induced vascular extravasation and angioedema in humans (12). These data suggest that ibuprofen may mitigate this aspect of the inflammatory response in COVID-19.

Widespread occlusion of pulmonary vasculature by microthrombi has been well demonstrated (14) with evidence that over $50 \%$ of patients dying of COVID-19 have pulmonary microthromboses. Thromboses are found not only in arterial vessels, but also in alveolar capillaries including in the absence of overt inflammation and ARDS (15). The anti-thrombotic effect of ibuprofen at low doses has been well known for decades, principally mediated via inhibition of platelet aggregation (16). Ibuprofen is a non-selective COX2 and COX1 inhibitor, an inhibitor of synthesis of thromboxane TBX2, a platelet pro-aggregant, vasoconstrictor, and pro-inflammatory cytokine, and an inhibitor of polymorphonuclear cell (PMN) release of reactive oxygen species and inflammatory mediators $(17,18,19,20)$.

We have previously reported that sodium-ibuprofenate is a membrane penetrating amphiphatic molecule with antibacterial properties in the presence of hypertonic saline, rendering the combination bactericidal for Pseudomonas aeruginosa and Burkholderia cepacia (21).

Viruses transiting cell membranes must navigate the actin-cytoskeleton of the target cell, a process regulated by Rho GTPase family members (22). Denison and colleagues demonstrated that SARSCoV-1 induces continuous macropinocytosis, and extensive filopodia outgrowth, and that inhibition of this process impaired virus replication (23). In a dengue virus model, activation of the GTPases Rac1 and Cdc42 and resulting filopodia growth was essential for virus entry and productive infection [24]. These authors noted that insulin and bradykinin, respectively, serve as agonists (activators) for these GTPases (this tantalizing side note may ultimately shed light on the observation that COVID19 is much more severe in patients with elevated insulin levels in the setting of type-2 diabetes). Ibuprofen inhibits Rho 
GTPase activation $[25,26]$ including Rac1b through a non-cyclooxygenase inhibiting pathway. Thus, additional activities of ibuprofen may have indirect antiviral properties relevant to SARS-CoV2, as well.

Given these potential benefits of NalHS in the context of SARS-CoV2 pneumonitis, we designed a compassionate-use program to offer this potentially beneficial therapy in the face of a pandemic pathogen against which few therapeutic options were available.

\section{Methods}

Ethical approval was obtained from the Institutional Independent Ethics Committees and district regulatory agencies of Cordoba and Mendoza Provinces for the compassionate use of Luarprofeno® (sodium ibuprofenate in hypertonic saline, or NalHS, for nebulization). The program was carried out in accordance with the principles of the Declaration of Helsinki for Buenos Aires. This study was registered with ClinicalTrials.gov, NCT04382768. All participating individuals were $\geq$ age 18 and provided written informed consent, obtained by the treating physician.

Luarprofeno® was manufactured under GMP conditions and provided by Quimica Luar SRL (QL, Cordoba, Argentina) in sterile vials, each containing $50 \mathrm{mg}$ of sodium ibuprofenate in $5 \mathrm{~mL}$ of hypertonic saline (comprised of $29 \mathrm{mg} \mathrm{NaCl} / \mathrm{mL}$, an 3\% solution). Each vial comprised a single dose. The threetimes daily dosing schema, each dose with $50 \mathrm{mg}$ dose, was selected based on preliminary observations $(21,28)$. The total daily administered ibuprofen dose was therefore $\leq 150 \mathrm{mg}$, recognizing that some drug is exhaled.

To submit a drug-request for patients, clinicians from Cordoba, Buenos Aires and Mendoza completed an assessment form with demographic and disease-status information about their patients. Approval of requests was reserved for hospitalized patients who had SARS-CoV-2 infection confirmed by reversetranscriptase-polymerase-chain-reaction assay and pneumonia. NalHS also was provided for a small number of patients ( $<5 \%$ ) with suspected COVID-19 infection based upon clinician assessment, bypassing the need for SARS-CoV-2 PCR diagnostics which were not consistently available in all regions at various times. Ibuprofen allergy was an exclusion criterion.

Each dose was administered using a standard piston-pump nebulizer, or via high flow oxygen or compressed ambient air, by face-mask. To reduce health care worker infection risk in the setting of nebulized therapy use, a novel containment hood was utilized with air venting via antiviral filters, designed to prevent particle aerosolization (note to reviewers, happy to provide supplemental data describing this system). All health care workers utilized PPE provided by their institutions. Each nebulized dose was administered over approximately 15 minutes, the time required to drain the liquid nebulization vessel. Treatment was initiated in participants with pulse oximetry saturation (SpO2) $\leq 94 \%$ or a need for oxygen support. Treatment was continued until SpO2 reached $\geq 94 \%$ while breathing ambient air for $>24$ hours or was stopped at clinician discretion or patient request. 
Supportive and standard of care (SOC) therapy was provided at the discretion of the clinician, as those therapies were available. As available, clinical status data collected by the clinician as part of standard care was to be shared with QL to evaluate program safety.

\section{Endpoint Assessments}

Patients were assessed daily during their hospitalization, from day 1 of treatment until discharge. These data were utilized to register patient clinical status based on the National Early Warning Score 2 for each day (29). All adverse events (AE) that were considered study-drug related were recorded. Vital signs were collected under routine hospital protocols every day between 07:00-11:00 am, before NalHS therapy. Outcomes in the smaller number of patients receiving MV support at the time of NalHS therapy initiation are presented separately in this manuscript. The average length of stay in hospitals (ALOS) and duration of treatment (DOT) were reported.

\section{Statistical analysis}

The analysis population included all patients with validated medical records who received compassionate use of NalHS therapy for at least 1 day. Most data analyses were descriptive. Summary measures included mean (SE), median (interquartile range), min and max values. Clinical status was evaluated through the determination of SpO2 measured by pulse oximetry, respiratory rates (RR) and NEWS2 scores over time, as well as ALOS and duration of supplemental oxygen therapy. The in-hospital mortality rate was determined. Treatment safety was assessed by tabulation of adverse events considered study drug related by treating physicians.

\section{Results}

\section{Overall Enrollment \& Baseline Characteristics}

A total of 383 patients were enrolled and received at least three doses $(50 \mathrm{mg})$ of NalHS (Figure 1); 253 from multiple hospitals in Cordoba Province, 95 from a single hospital in Buenos Aires and 35 from multiple hospitals in Mendoza Province. At Baseline (before initiation of NalHS therapy) 327 were not receiving mechanical ventilation (MV) while 56 patients (14.6\%) were receiving MV. Outcome measures are presented for these two aggregate groups separately. Among patients not receiving MV at baseline $(n=327)$, mean age was 59 and the majority (62.5\%) were male (Table 1). Prior diagnoses of diabetes, cardiovascular disease including hypertension, and chronic lung disease were noted in $22 \%, 44 \%$ and $14 \%$ of patients, respectively. Baseline SpO2 was $91 \%$, with $75 \%$ of patients on supplemental oxygen at the time of measurement (baseline). The mean and median NEWS 2 score at baseline for this group was 7.0.

Among patients receiving MV at baseline (n-56), mean age was 60.6 and the majority were male (Table 1). Prior diagnoses of diabetes, cardiovascular disease including hypertension and chronic lung disease was noted in $28.6 \%, 46.6 \%$ and $23.2 \%$ of patients, respectively. Baseline SpO2 was $90 \pm 0.8$, and $100 \%$ 
were on supplemental oxygen at the time of measurement (baseline). The mean and median NEWS 2 score at baseline for this group was 8.7 and 8.0.

\section{Clinical Outcomes}

NEWS 2 Scores: The mean daily NEWS 2 score for patients not on MV at Baseline is presented in Figure 2. Progressive improvement over Days 1 through 6 to a mean NEWS2 score of $<4$ is evident in the group as a whole. For a subset of patients $(n=60)$, NEWS 2 scores were available for a time period of 1 to 3 days prior to initiation of NalHS therapy. Strikingly, this subgroup of patients demonstrated progressive clinical deterioration indicated by worsening NEWS2 scores prior to initiation of NalHS therapy, with peak NEWS 2 score evident on the day of (but prior to) therapy initiation. This clinical course reversed dramatically coinciding with initiation of NalHS therapy. In Figure 2, data for patients enrolled from each province are graphed separately demonstrating a range in baseline disease severity.

\section{Respiratory Rates:}

The mean daily respiratory rate (RR) for patients not on MV at baseline are presented in Figure 3A. Consistent with the observation of NEWS2 scores over time, following initiation of NalHS there was steady improvement in mean RR for this group, and for the subset with pre-ibuprofen therapy data collection, a trend of increasing (worsening) RR despite the addition of supplemental oxygen is clear, with immediate improvement after NalHS initiation. Overall, mean RR for the entire group had normalized ( $\leq$ 20 breaths/minute) by Day- 6 , with decreasing numbers of patients on supplemental oxygen during this time period.

Oxygen Saturation: Mean daily pulse oximetry oxygen saturation for patients not on MV at Baseline is presented in Figure 3B. As was observed with RR and NEWS2 scores, among patients with available data prior to initiation of therapy with NalHS, there was daily deterioration in oxygen saturation (despite increasing use of supplemental oxygen), which reversed acutely with initiation of therapy. By Day 6 , mean SpO2 was $>94 \%$, remaining at this level with decreasing numbers of patients on supplemental oxygen and discharge from hospital of increasing numbers of patients. Importantly, this figure reflects the SpO2 status of patients still hospitalized; as patients improved, they were discharged, creating a data bias towards flattening of the improvement curve (or worsening). Indeed, this phenomenon was observed in the Cordoba subset of patients at Days 9-10, but for the entire study population, stable improvement of oxygenation is observed.

Length of Hospital Stay (LOS) \& Duration of Therapy (DOT): Among patients not on MV at baseline, the average LOS was $11.5 \pm 0.3$ days (Table 1 ), with DOT of $9.0 \pm 0.2$ days. In this group, 25 patients never required oxygen supplementation; among those receiving oxygen therapy $(n=302)$, the mean duration of oxygen supplementation was 6 days, with a median duration of 5 days. For the 35 patients who progressed to death (10.7\%), the average LOS was $13 \pm 1.2$ days. Among patients on MV at baseline, the average LOS was $15.5 \pm 1.4$ days (Table 2), with DOT of $10.5 \pm 0.7$ days. For the 11 patients who progressed to death in hospital (19.6\%), the ALOS was $13.5 \pm 3.3$ days. 
In Hospital Mortality Rates: Overall in-hospital mortality among patients receiving NalHS was $12.0 \%$ (46/383). At the time of data collection 28 patients remained in hospital (some for social reasons despite returning to clinical stability). Mortality among patients not on $\mathrm{MV}$ at baseline was $10.7 \%$, and for those on MV at baseline, $19.6 \%$ (Figure 1). For the subset of patients not on MV at baseline $(n=327)$, mortality rates were influenced by age and baseline oxygenation status (Tables 2 and 3 ). Among patients of age $\leq$ 60 (Table 2), overall mortality was $3.4 \%(6 / 174)$, occurring in 5 patients who presented with baseline $\mathrm{SpO} 2 \leq 90 \%$, and in 1 who presented with normal oxygenation but died with sepsis (an immunosuppressed renal transplant recipient). Among patients > age 60 (Table 3), overall mortality was $19.0 \%(29 / 153)$, the majority of deaths occurring in patients presenting with SpO2 <90\% $(19 / 29,66 \%)$.

Concomitant medications: SOC medications administered to the majority of patients included: dexamethasone $6 \mathrm{mg}$ qd, (79.4\%), enoxaparin $40 \mathrm{mg} \mathrm{sc}$ qd (81.2\%), and the combination of clarithromycin $500 \mathrm{mg}$ bid and ampicillin-sulbactam $3.0 \mathrm{gr}$ tid (63.2\%). A small number of patients received convalescent plasma, ivermectin or hydroxychloroquine.

Adverse Events: No serious AE events were considered directly related to NalHS. Patients reported mild to moderate cough during the first doses of NalHS and salty taste. Bradycardia associated with improved SpO2 was observed in some patients. Three episodes of epistaxis were reported. One patient reported ibuprofen allergy, another claustrophobic reaction due to the hood and another patient developed a cough that was considered secondary to nebulization therapy, these three discontinued the medication permanently.

\section{Discussion}

This report describes clinical outcomes in a moderate size cohort of patients who were severely ill with Covid-19 and treated with NalHS under a compassionate use protocol. As there was no comparator arm or randomization, the data can only be considered hypothesis-generating. Despite this limitation, multiple lines of evidence suggest that nebulized NalHS, may be a useful therapeutic tool for the treatment of moderate-to-severe COVID-19, and is worthy of deeper examination in a randomized controlled trial (RCT) context.

The course of multiple critical patient outcome parameters (NEWS2 Scores, RR and SpO2) improved rapidly following the initiation of NalHS. For the subset of 60 patients for whom these data were available prior to treatment initiation, an acute reversal in trend was observed with a decrease in mean respiratory rate coinciding with improving oxygen saturation and NEWS2 scores immediately following initiation of therapy. By Day- 6 following initiation of therapy, mean NEWS2 scores improved to $<4$, pulse oximetry had improved to $\geq 94 \%$ with diminishing supplemental oxygen needs, and respiratory rates had returned to normal. These observations are consistent with the conceptual interpretation that pulmonary delivery of NalHS diminished bradykinin-storm physiology, likely reduced local prostaglandin and thromboxane synthesis and acutely improved the ventilation-perfusion mismatch which is a hallmark of this disease as we hypothesized above. 
The overall mortality rate of $10.7 \%$ observed in this study in patients not on $\mathrm{MV}$ at baseline compares favorably with recently published data (Supplemental Table 1). Mortality was 2-fold higher (19.6\%) among patients on MV at baseline (versus $10.7 \%$ overall in non-intubated patients), and among nonintubated patients, mortality was strikingly higher among patients > age $60(19.0 \%)$ versus those age 60 or younger (3.4\%).

In the published series, mortality rates among patients with severe COVID-19 and on MV at baseline of $13 \%$ (30) and ranging up to $41 \%$ and higher (31) have been reported. While a cross-study comparison of outcomes between trials can only be considered a range-finding exercise for a host of reason (particularly in the context of continual improvements in care), it is noteworthy that mortality among patients receiving dexamethasone in the RECOVERY trial was over 29\% (noting that most patients in this NalHS trial received dexamethasone as part of SOC). Recently, results from an Argentinian randomized controlled trial, evaluating treatment with convalescent plasma versus placebo were published (4). This trial, which excluded patients on MV at screening, reported an overall 28-day mortality rate of approximately $11 \%$, remarkably similar to the in-hospital mortality rate of $10.7 \%$ observed among patients not on MV in the present study.

Importantly, our compassionate use protocol did not exclude patients with profound and confounding major diseases, which would be expected to have increased overall mortality, in comparison. For instance, among patients enrolled in Buenos Aires, three of the nine deceased patients were admitted with lifethreatening illnesses (metastatic pancreatic cancer, bacterial sepsis in a renal transplant recipient, and perforation of the appendix with peritonitis) and subsequently succumbed to these illnesses with concomitant COVID-19 infection. Accordingly, similar mortality rates in the present study point to the possibility of treatment benefit of NalHS. In this vein of reasoning, despite an effort through exclusion criteria to avoid enrollment of patients in imminent need of ICU admission and MV in the convalescent plasma trial, a total of $85 / 333$ patients $(25.5 \%)$ who were not intubated at baseline ultimately required this intervention. In the present study, clinicians had no restraint preventing enrollment of such patients, yet a smaller proportion (18.9\%) of those not on MV at baseline ultimately required this intervention. Finally, in the convalescent plasma trial (4), the time from intervention to hospital discharge for plasma and placebo treatment was 13 and 12 days, respectively; in our study, the ALOS was $11.5 \pm 0.3$ and $15.5 \pm$ 1.4 days for non-ventilated and ventilated groups, respectively. These may be meaningful differences, but of course this can only be assessed in a future RCT. In light of questions raised early in the pandemic regarding the safety of non-steroidal anti-inflammatory drug use in COVID-19 patients, this cross-study comparison should reassure that inhaled ibuprofen does not create excess risk when added to SOC interventions.

The mechanism by which NalHS induces an apparent rapid improvement in oxygenation and other vital signs in COVID-19 patients is unknown but multiple potential mechanisms can be evoked. These include the classical anti-inflammatory activity mediated by inhibition of prostaglandin and bradykinin synthesis, inhibition of thromboxane's platelet aggregation and reduction of reactive oxygen species by polymorphonuclear cells as previously observed (28). A potential benefit of nebulized hypertonic saline, 
recognized to be a mucolytic agent and used for this purpose in the treatment of cystic fibrosis and bronchiectasis, should not be overlooked. Finally, both direct antiviral and antibacterial activity, as well as potential indirect antiviral activity through inhibition of RhoGTPase activity may have contributed.

This program of course has limitations. First, this is a description of a non-randomized observational cohort receiving compassionate use therapy, and causal relationships to outcomes cannot be proven. Second, only hospitalized adults were evaluated, and the findings may not be generalizable to other populations, including non-hospitalized patients. Third, given the nature of the study, many critical parameters were not prospectively evaluated (pharmacovigilance, FiO2). Finally, only a single dosing regimen of ibuprofen was studied. Despite these limitations, encouraging efficacy trends emerged.

\section{Conclusion}

The compassionate use of sodium ibuprofenate administered by nebulization in hypertonic saline appeared to be safe and well-tolerated in this cohort with varying severity of illness. Average length of stay and mortality rates compared favorably with published outcomes from randomized controlled trials. Reversal of deleterious physiologic trends, overall, coincided with the initiation of treatment. NalHS appears to be a promising therapy for treatment of COVID-19 pneumonitis, warranting further evaluation in randomized controlled trials.

\section{Abbreviations}

$\mathrm{AE}$

adverse events

ALOS

average length of stay

ARDS

acute respiratory distress syndrome

DOT

duration of treatment

ICU

INtensive Care Unit

LOT

length of treatment

MV

mechanical ventilation

NalHS

Sodium-ibuprofenate in hypertonic saline

QL

Quimica Luar SRL

RCT 
randomized controlled trial

RR

respiratory rates

SOC

Standard of care

$\mathrm{SpO} 2$

Oxygen pulse saturation

\section{Declarations}

\section{Availability of data and materials}

The datasets during and/or analysed during the current study is available from the corresponding author on reasonable request.

\section{Acknowledgments}

We thank Drs. Ernesto Jakob and Flavio Lipari (infectologists), Drs. Julio Bartoli and Ignacio Ledesma (intensivists), for assistance during the protocol development.

\section{Funding}

This work was supported by funds from the Quimica Luar SRL, who provided NalHS under compassionate use. This funding body had no role in: the design of the study; the collection, analysis, or interpretation of data; and the writing of the manuscript.

\section{Author information}

Dr. Salva and Dr. Doreski contributed equally to this article

\section{Author contributions}

Conceptualization: PAD, GA, LAA, NMR, DMB and NHG. Data curation: SEM, MNC, DJP, HAP, NHG. Formal analysis: PAD, SEM, MNC, DJP, HAP, DMB, NHG. Funding acquisition: LAA, NMR and GIK. Investigation: OS, CSG, DCQ, LGG, GA, EC, JLTD, GDB, JOF, MAM, LEPC, FF, HAP, MAQ, JASM, CMP, Methodology: LAA, NMR, DMB, NHG. Project administration: LAA, NMR, GIK. Resources: LAA and NMR. Supervision: LAA, NMR, NHG. Software: HAP. Validation: OS, PAD, NHG. Visualization: NHG. Writing-original draft: NHG. Writing-review and editing: PAD, DCQ, LAA, NMR, DMB, NHG.

\section{Ethics declarations}

\section{Ethics approval and consent to participate}


Ethical approval was obtained from the Institutional Independent Ethics Committees and district regulatory agencies of Cordoba and Mendoza Provinces for the compassionate use of Luarprofeno® (sodium ibuprofenate in hypertonic saline, or NaIHS, for nebulization). The program was carried out in accordance with the principles of the Declaration of Helsinki for Buenos Aires. All participating individuals were $\geq$ age 18 and provided written informed consent, obtained by the treating physician.

\section{Consent for publication}

Not applicable.

\section{Competing interests}

The authors declare that they have no competing interests.

\section{Conflict of interest}

The authors have declared that no conflict of interest exists.

\section{References}

1. https://www.who.int/emergencies/diseases/novel-coronavirus-2019

2. RECOVERY Collaborative Group. Dexamethasone in hospitalized patients with Covid-19-Preliminary report. New Engl J Med 2020 doi: 10.1056/NEJMoa2021436.

3. WHO Solidarity Trial Consortium, Pan H, Peto R, Henao-Restrepo AM, Preziosi MP, Sathiyamoorthy V, Abdool Karim Q, Alejandria MM, Hernández García C, Kieny MP, Malekzadeh R, Murthy S, Reddy KS, Roses Periago M, Abi Hanna P, Ader F, Al-Bader AM, Alhasawi A, Allum E, Alotaibi A, Alvarez-Moreno CA, Appadoo S, Asiri A, Aukrust P, Barratt-Due A, Bellani S, Branca M, Cappel-Porter HBC, Cerrato N, Chow TS, Como N, Eustace J, García PJ, Godbole S, Gotuzzo E, Griskevicius L, Hamra R, Hassan M, Hassany M, Hutton D, Irmansyah I, Jancoriene L, Kirwan J, Kumar S, Lennon P, Lopardo G, Lydon P, Magrini N, Maguire T, Manevska S, Manuel O, McGinty S, Medina MT, Mesa Rubio ML, MirandaMontoya MC, Nel J, Nunes EP, Perola M, Portolés A, Rasmin MR, Raza A, Rees H, Reges PPS, Rogers CA, Salami K, Salvadori MI, Sinani N, Sterne JAC, Stevanovikj M, Tacconelli E, Tikkinen KAO, Trelle S, Zaid H, Røttingen JA, Swaminathan S. Repurposed Antiviral Drugs for Covid-19 - Interim WHO Solidarity Trial Results. N Engl J Med. 2020 Dec 2. doi: 10.1056/NEJMoa2023184. Epub ahead of print. PMID: 33264556.

4. Simonovich VA, Burgos Pratx LD, Scibona P, Beruto MV, Vallone MG, Vázquez C, Savoy N, Giunta DH, Pérez LG, Sánchez MDL, Gamarnik AV, Ojeda DS, Santoro DM, Camino PJ, Antelo S, Rainero K, Vidiella GP, Miyazaki EA, Cornistein W, Trabadelo OA, Ross FM, Spotti M, Funtowicz G, Scordo WE, Losso MH, Ferniot I, Pardo PE, Rodriguez E, Rucci P, Pasquali J, Fuentes NA, Esperatti M, Speroni GA, Nannini EC, Matteaccio A, Michelangelo HG, Follmann D, Lane HC, Belloso WH; PlasmAr Study Group. A Randomized Trial of Convalescent Plasma in Covid-19 Severe Pneumonia. N Engl J Med. 2020 Nov 24. doi: 10.1056/NEJMoa2031304. Epub ahead of print. PMID: 33232588. 
5. Cao B, Wnag Y, Wen D, Liu W, Wang J, Fan G, Ruan L, Song B et al A trial of lopinavir ritonavir in adults hospitalized with severe Covid-19. N Engl J Med 2020;382:1787-99 doi:

10.1056/NEJMoa2001282.

6. Cavalcanti AB, Zampieri FG, Rosa RG, Azevedo LCP, Veiga VC, Avezum A, Damiani LP, Marcadenti A et al. Hydroxychloroquine with or without azithromycin in mild-tomoderate Covid-19. New Engl J Med 2020 doi: 10.1056/NEJMoa2019014.

7. Stone JH, Frigault MJ, Serling-Boyd NJ, Fernandes AD, Harvey L, Foulkes AS et al for the BACC Bay Tocilizumab Trial Investigators. Efficacy of tocilizumab in patients hospitalized with Covid-19. New Eng J Med 2020 DOI: 10.1056/NEJMoa2028836.

8. Shi H, Han X, Jiang N, Cao Y, Alwalid O, Gu J, Fan Y, Zheng C. Radiological findings from 81 patients with COVID-19 pneumonia in Wuhan, China: a descriptive study. Lancet Infect Dis. 2020 Apr;20(4):425-434. doi: 10.1016/S1473-3099(20)30086-4. Epub 2020 Feb 24. PMID: 32105637; PMCID: PMC7159053.

9. Ackermann M, Verleden S, Kuehnel M, Haverich A, Welte T, Laenger F, Vanstapel A et al Pulmonary vascular endothelialitis, thrombosis and angiogenesis in Covid-19. N Eng J Med 2020; 383:120128 doi: 10.1056/NEJMoa2015432.

10. Garvin MR, Alvarez C, Miller JI, Prates ET, Walker AM, Amos BK, Mast AE, Justice A, Aronow B, Jacobson D. A mechanistic model and therapeutic interventions for COVID-19 involving a RASmediated bradykinin storm. eLife 2020; 9:e59177 doi:10.7554/eLife.59177.

11. Baraniuk JN, Lundgren JD, Mizoguchi H, Peden D, Gawin A, Merida M, Shelhamer JH, Kaliner MA. Bradykinin and respiratory mucous membranes. Analysis of bradykinin binding site distribution and secretory responses in vitro and in vivo. Am Rev Respir Dis. 1990 Mar;141(3):706-14. doi: 10.1164/ajrccm/141.3.706. PMID: 2178531.

12. Gholamreza-Fahimi E, bisha M, hanh J, Straben U, Krybus M, Khosravani F, Hoffman TK, Hohlfeld T et al. Cycooxygenase activity in bradykinin-induced dermal extravasation. A study in mice and humans. Biomedicine \& Pharmacology 2020 123: 109797 doi:10.1016/j.biopha.2019.109797.

13. Brito-Azevedo, A, Pinto, EC, Corrêa, GAdCP, Bouskela, E. SARS-CoV-2 infection causes pulmonary shunt by vasodilatation. J Med Virol. 2020; 1- 3. doi:10.1002/jmv.26342.

14. McFadyen, James D et al. "The Emerging Threat of (Micro)Thrombosis in COVID-19 and Its Therapeutic Implications.” Circulation research vol. 127,4 (2020): 571-587. doi:10.1161/CIRCRESAHA.120.317447

15. Magro C., et al. Complement associated microvascular injury and thrombosis in the pathogenesis of severe COVID-19 infection: a report of five cases. Transl Res (2020).

16. Mclntyre BA, Philp RB, Inwood MJ. Effect of ibuprofen on platelet function in normal subjects and hemophiliac patients. Clin Pharmacol Ther. 1978 Nov;24(5):616-21. doi:10.1002/cpt1978245616. PMID: 699486.

17. Walsh SW. Low-dose aspirin: treatment for the imbalance of increased thromboxane and decreased prostacyclin in preeclampsia. Am J Perinatol. 1989 Apr;6(2):124-32. doi: 10.1055/s-2007-999562. 
PMID: 2653334.

18. Hammock BD, Wang W, Gilligan MM, Panigrahy D. Eicosanoids: the Overlooked Storm in COVID-19?, The American Journal of Pathology (2020), doi:10.1016/j.ajpath.2020.06.010.

19. Serhan CN. Systems approach to inflammation resolution: identification of novel antiinflammatory and pro-resolving mediators. J Thromb Haemost. 2009 Jul;7 Suppl 1:44-8. doi: 10.1111/j.15387836.2009.03396.x. PMID: 19630766.

20. Das UN. Can Bioactive Lipids Inactivate Coronavirus (COVID-19)? Arch Med Res. 2020 Apr;51(3):282286. doi: 10.1016/j.arcmed.2020.03.004. Epub 2020 Mar 27. PMID: 32229155; PMCID: PMC7270578.

21. Munoz AJ, Alasino RV, Garro AG, Heredia V, Garcia NH, Cremonezzi DC, Beltramo DM High concentrations of sodium chloride improve microbicidal activity of ibuprofen against common Cystic Fibrosis pathogens. Pharmaceuticals (Basel) 2018 11(2)47 doi: 10.3390/ph11020047.

22. Swain T, Dittmar MT. CDC42 use in viral cell entry processes by RNA viruses. Viruses 2015, 7, 65266536; doi: 10.3390/v7122955.

23. Freeman MC, Peek CT, Becker MM, Smith EC, Denison MR. Coronavirus induce entry independent, continuous macropinocytosis. mBio 2014: 5(4) e01340-14. doi: 101128/mBio01240-14.

24. Zamudio-Meza H, Castillo-Alvarez A, Gonzalez-Bonilla C, Mezra I. Cross-talk between Rac1 and Cdc42 GTPases regulates formation of filopodia required for dengue virus type-2 entry into HMEC-1 cells. J Gen Virol 2009, 9-0, 2902-2911. doi 10.1088/vir.0.014159-0

25. Zhou Y, Su Y, Li B, et al. Nonsteroidal anti-inflammatory drugs can lower amyloidogenic $A \beta 42$ by inhibiting Rho. Science 2003:302; 1215-1217.

26. Dill J, Patel AR, Yang R, Bachoo R, Powell M, Li S. A molecular mechanism for ibuprofen mediated RhoA inhibition in neurons. Journal of Neuroscience 2010:30(3):963-972.

27. Matos P, Kotelevets L, Goncalves V, Henriques A, Zerbib P, Moyer MP, Chastre E, Jordan P. Ibuprofen inhibits colitis-induced overexpression of tumor-related Rac1b. Neoplasia 2013 15(1)102-111.

28. García NH, Porta DJ, Alasino RV, Muñoz SE, Beltramo DM. Ibuprofen, a traditional drug that may impact the course of COVID-19 new effective formulation in nebulizable solution. Med Hypotheses. 2020 Nov;144:110079. doi: 10.1016/j.mehy.2020.110079. Epub 2020 Jul 7. PMID: 32758897; PMCID: PMC7340056.

29. Royal College of Physicians. National Early Warning Score (NEWS) 2. 2017 https://www.rcplondon.ac.uk/projects/outputs/national-early-warning-score-news-2.

30. Beigel JH, Tomashek KM, Dodd LE, Mehta AK, Zingman BS, Kalil AC, Hohmann E, Chu HY et al. Remdesivir for the treatment of Covid-19-Preliminary Report. New Engl J Med 2020 doi:10.1056/NEJMoa2007764.

31. RECOVERY Collaborative Group. Dexamethasone in hospitalized patients with Covid-19- Preliminary report. New Engl J Med 2020 doi: 10.1056/NEJMoa2021436. 


\section{Tables}

Table 1: Baseline Characteristics of 383 Hospitalized COVID19 Patients who Received Compassionate Use Nebulized Ibuprofen Therapy

\begin{tabular}{|c|c|c|}
\hline Baseline Characteristic & $\begin{array}{l}\text { Not on Mechanical } \\
\text { Ventilation at Baseline } \\
(n=327)\end{array}$ & $\begin{array}{l}\text { On Mechanical } \\
\text { Ventilation at Baseline } \\
(n=56)\end{array}$ \\
\hline Mean Age (in years) & $59 \pm 1.0$ & $60.6 \pm 2.2$ \\
\hline Median & $59(20)$ & $63.5(19.5)$ \\
\hline Range & $26-91$ & $19-95$ \\
\hline Female Patients & $123(37.5 \%)$ & $17(30.4 \%)$ \\
\hline \multicolumn{3}{|l|}{ Risk Factors for Disease Severity } \\
\hline Has any Risk Factor* & $55.7 \%$ & $69.6 \%$ \\
\hline Diabetes & $73(22 \%)$ & $16(28.6 \%)$ \\
\hline HTN) Cardiovascular Disease (includes & $13(44 \%)$ & $25(46.6 \%)$ \\
\hline Chronic lung disease & $46(14 \%)$ & $13(23.2 \%)$ \\
\hline Proportion Receiving Supplemental $\mathrm{O}_{2}$, Day- 1 & $244(75 \%)$ & $56(100 \%)$ \\
\hline $\begin{array}{l}\text { Mean Baseline } \mathrm{O}_{2} \text { Saturation (pulse } \\
\text { oximetry) }\end{array}$ & $91.0 \pm 0.3 \%$ & $90.0 \pm 0.8 \%$ \\
\hline $\begin{array}{l}\text { Mean Baseline Respiratory Rate } \\
\text { (breaths/min) }\end{array}$ & $23 \pm 0.3$ & $23 \pm 0.8$ \\
\hline \multicolumn{3}{|l|}{ Baseline NEWS2 Score * } \\
\hline Mean & $7.0 \pm 0.1$ & $8.7 \pm 0.4$ \\
\hline Median & $7(4)$ & $8(3)$ \\
\hline Range & $1-15$ & $2-15$ \\
\hline $\begin{array}{l}\text { Proportion receiving dexamethasone therapy } \\
\text { (at some point during treatment course) }\end{array}$ & $257(78.4 \%)$ & $45(80.4 \%)$ \\
\hline Mean Length of Hospitalization (Days) & $11.5 \pm 0.3$ & $15.5 \pm 1.4$ \\
\hline Mean Duration of Ibuprofen therapy (Days)* & $9.0 \pm 0.2$ & $10.5 \pm 0.7$ \\
\hline $\begin{array}{l}\text { Mean time in hospital to death, deceased } \\
\text { patients }(n)\end{array}$ & $13 \pm 1.2(n=35)$ & $13.5 \pm 3.3(n=11)$ \\
\hline
\end{tabular}


Table 2: Mortality rate by age and baseline oxygen saturation. Patients age $\leq 60$

\begin{tabular}{|c|c|c|c|c|c|}
\hline \multirow[b]{2}{*}{ Final SpO2 } & \multicolumn{5}{|c|}{ Baseline SpO2 } \\
\hline & $95-99 \%$ & $91-94 \%$ & $86-90 \%$ & $<86 \%$ & Total \\
\hline \multirow[t]{2}{*}{ Normal (\%) } & 28 (16.38) & $50(29.25)$ & 57 (33.33) & $8(4.68)$ & 143 \\
\hline & & & & & (83.64) \\
\hline \multirow[t]{2}{*}{$91-94 \%$} & $4(2.34)$ & $8(4.68)$ & $11(6.44)$ & $1(0.58)$ & 24 \\
\hline & & & & & (14.04) \\
\hline $86-90 \%$ & 0 & $1(0.58)$ & 0 & $1(0.58)$ & $2(1.16)$ \\
\hline$<86 \%$ & 0 & 0 & $2(1.16)$ & 0 & $2(1.16)$ \\
\hline Discharges & $31(18.14)$ & 57 (33.33) & $65(38.02)$ & $9(5.27)$ & $162(94.76)$ \\
\hline Death & 0 & 0 & $4(2.34)$ & $1(0.58)$ & $5(2.92)$ \\
\hline Remain Hospitalized & $1(0.58)$ & $2(1.16)$ & $1(0.58)$ & 0 & $4(2.32)$ \\
\hline Total (\%) & 32 (18.72) & 59 (34.51 & 70 (40.93) & $10(5.84$ & 171(100) \\
\hline
\end{tabular}

Table 3: Mortality rate by age and baseline oxygen saturation. Patients age $\$ 60$

\begin{tabular}{|llllll|}
\hline \multirow{2}{*}{ Final SpO2 } & \multicolumn{2}{l}{ Baseline SpO2 } & & \\
\cline { 2 - 6 } & $95-99 \%$ & $91-94 \%$ & $86-90 \%$ & $<86 \%$ & Total \\
\hline Normal & $14(9.28)$ & $28(18.55)$ & $41(27.15)$ & $5(3.31)$ & $88(58.29)$ \\
\hline $91-94 \%$ & $4(2.65)$ & $12(7.95)$ & $25(16.56)$ & $1(0.66)$ & $42(27.82)$ \\
\hline $86-90 \%$ & $1(0.66)$ & $4(2.65)$ & $6(3.97)$ & $2(1.32)$ & $13(8.6)$ \\
\hline$<86 \%$ & 0 & 0 & $6(3.97)$ & $2(1.32)$ & $8(5.29)$ \\
\hline Discharges (\%) & $16(10.6)$ & $35(23.17)$ & $54(35.76)$ & $5(3.31)$ & $110(72.84)$ \\
\hline Death (\%) & 0 & $9(5.96)$ & $16(10.6)$ & $3(1.99)$ & $28(18.55)$ \\
\hline Remain Hospitalized (\%) & $3(1.99)$ & 0 & $8(5.3)$ & $2(1.32)$ & $13(8.61)$ \\
\hline Total & $19(12.59)$ & $44(29.13)$ & $78(51.66)$ & $10(6.62)$ & $151(100)$ \\
\hline
\end{tabular}




\section{Participant enrollment, Compassive-use nebulized ibuprofen therapy for moderate to severe COVID-19 in Argentina}

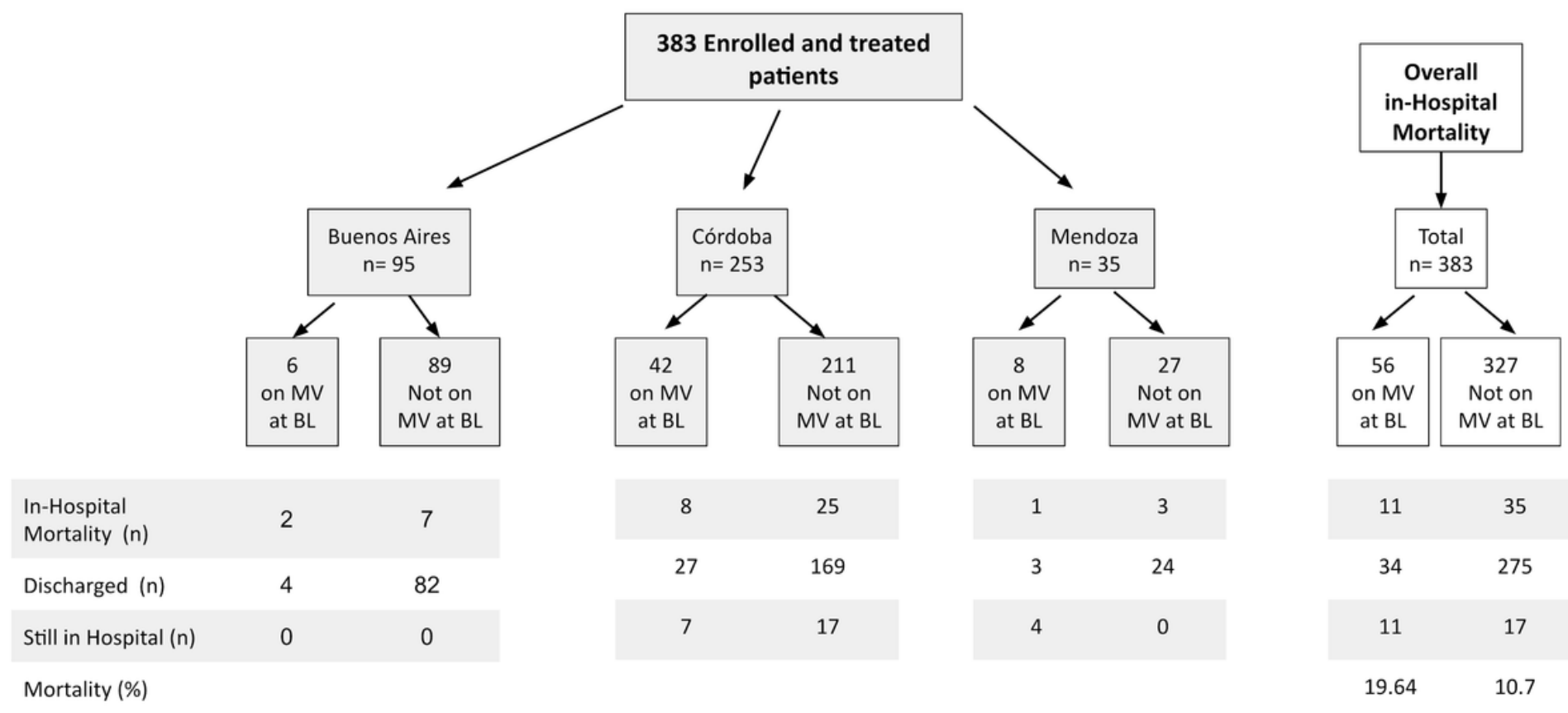

\section{Figure 1}

Participant Enrollment, Compassionate-Use Nebulized Ibuprofen Therapy for Moderate to Severe COVID19 in Argentina

Cumulative \# of patients Hospitalized Cumulative \# of patients Discharged Cumulative \# of patients Deceased

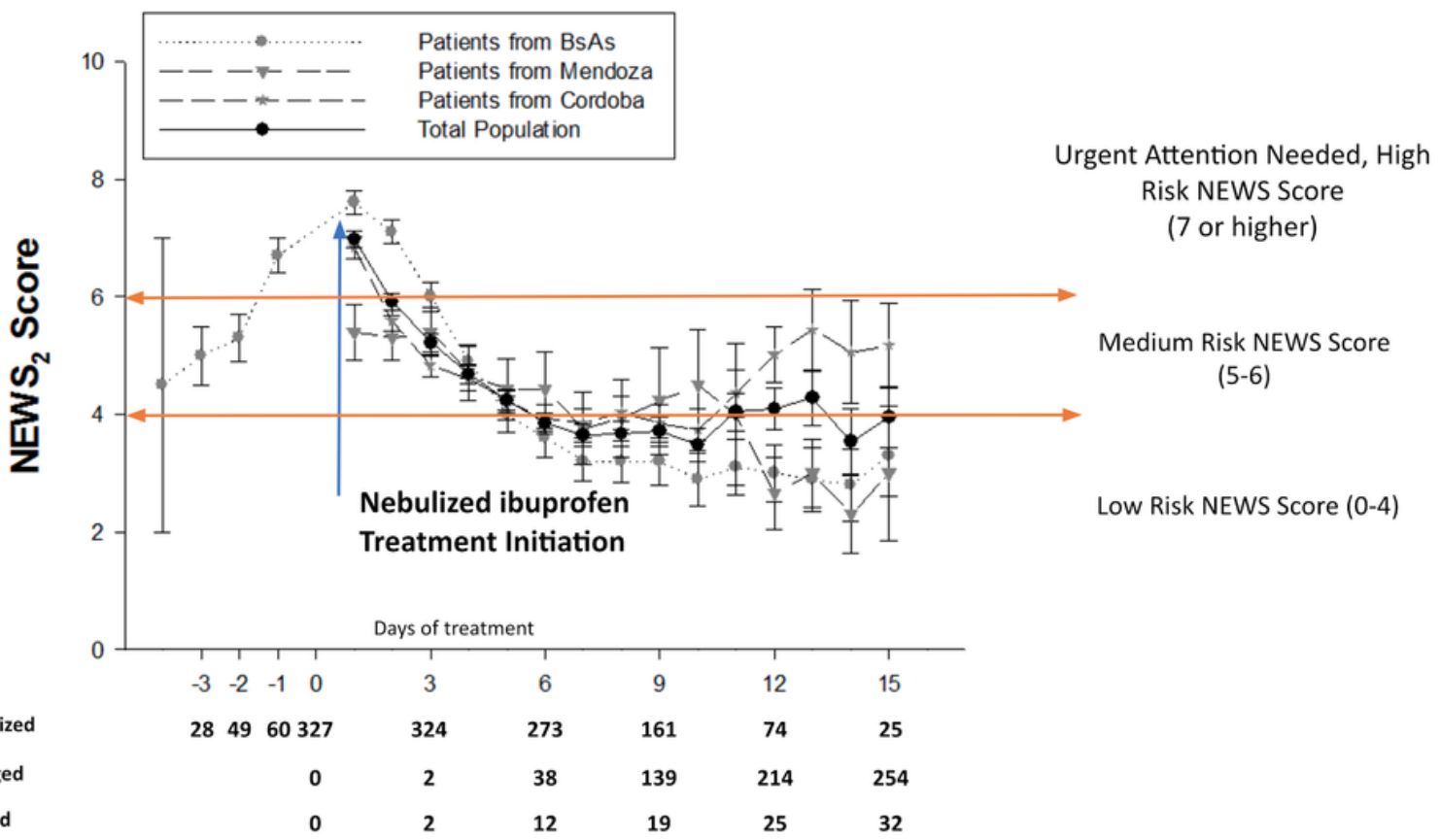


Figure 2

NEWS2 Score among Patients not on Mechanical Ventilation at Baseline who Received Nebulized Ibuprofen

A

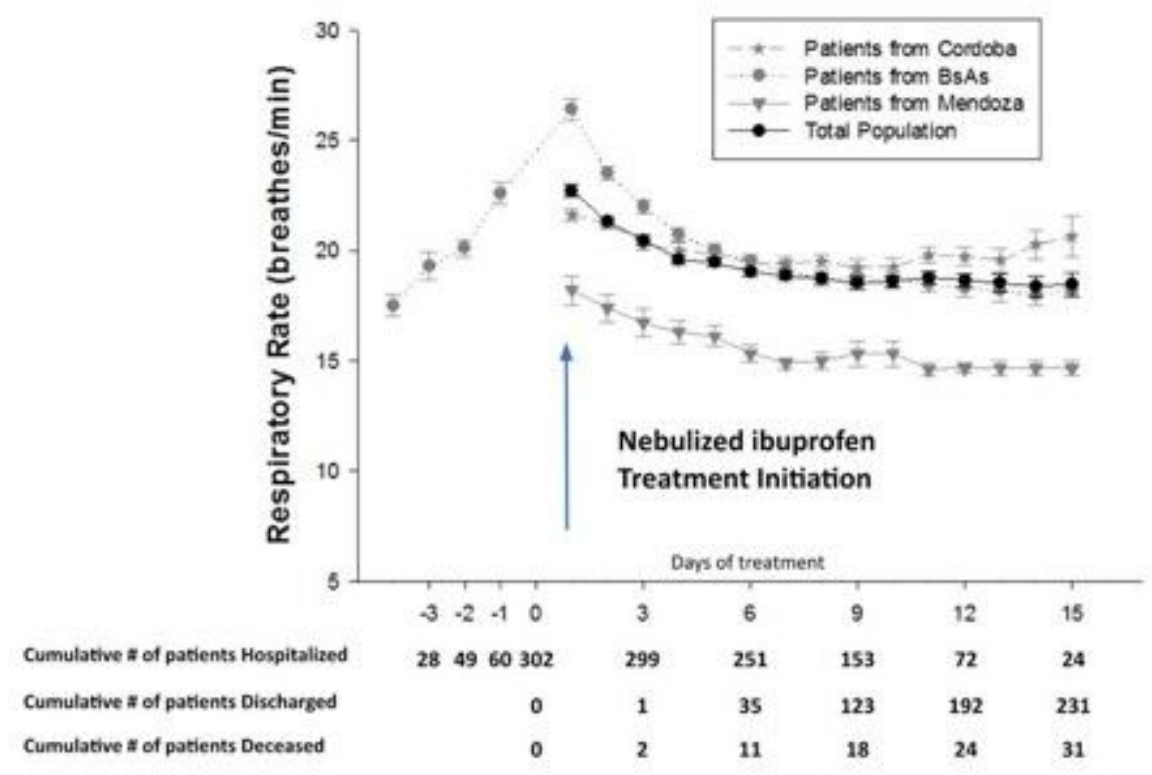

B

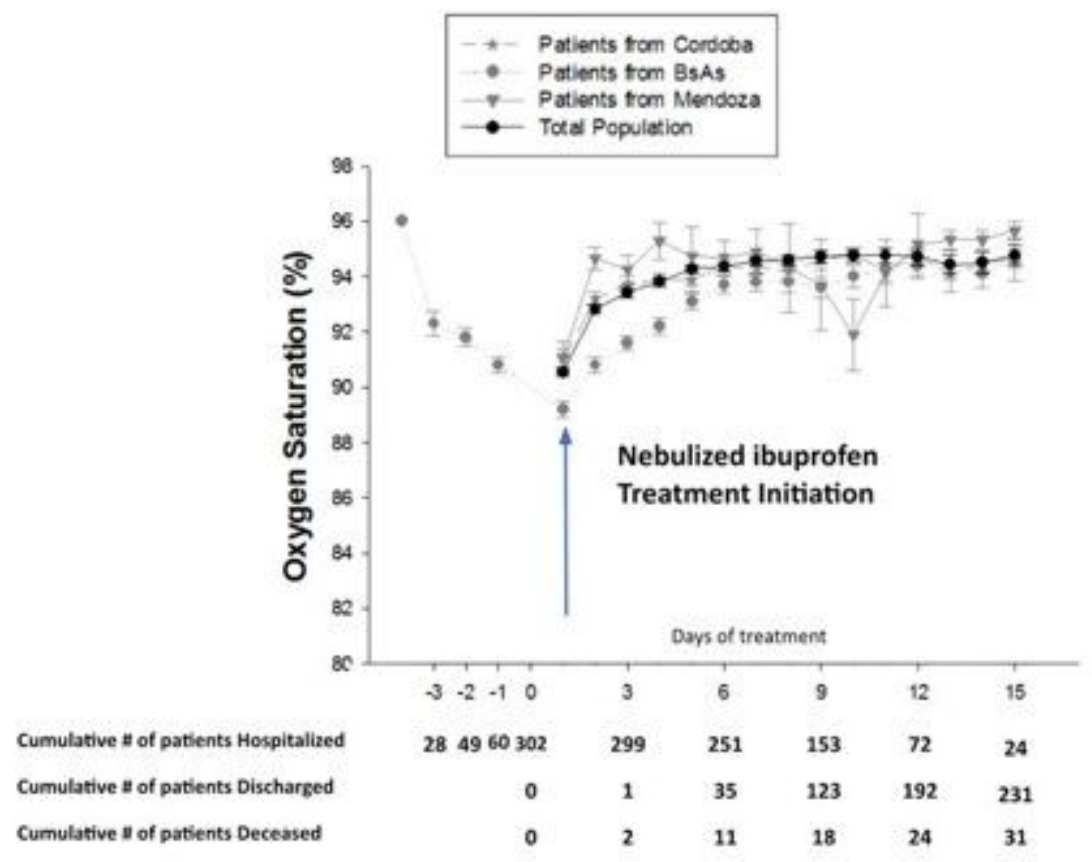

Figure 3

Respiratory Rate (A) and Oxygen Saturation (B) among Patients not on Mechanical Ventilation at Baseline who Received Nebulized Ibuprofen (25 patients were censored because never received oxygen 
supplementation).

\section{Supplementary Files}

This is a list of supplementary files associated with this preprint. Click to download.

- SupplementalTable1.docx 